\title{
Hydroxyurea-induced ulcers on the leg
}

Joachim Dissemond MD, Andreas Körber MD

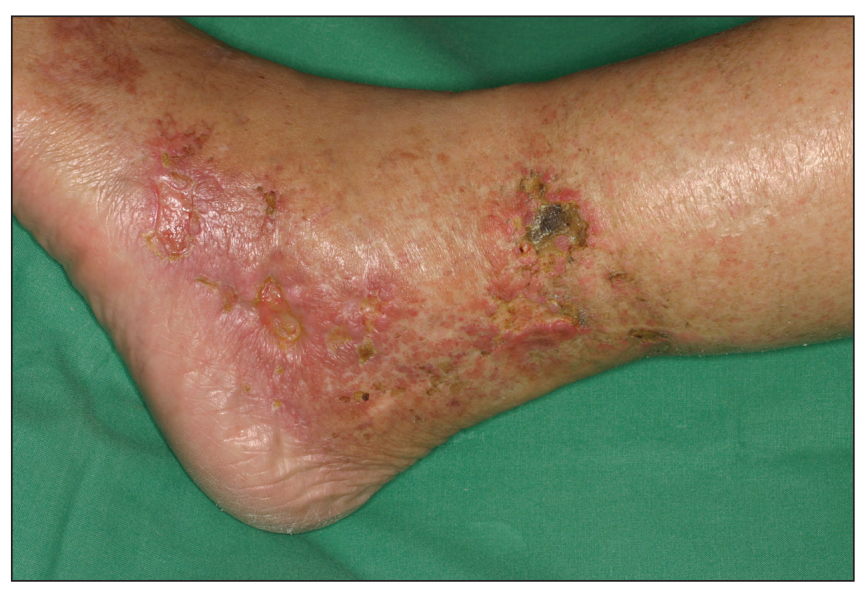

Figure 1: Hydroxyurea-induced ulcers on the leg of a 73-year-old man with chronic myelogenous leukemia. 73-year-old man with a history of chronic myelogenous leukemia presented with multiple ulcers on both legs (Figure 1). The ulcers were extremely painful. The patient had no history of diabetes or hypertension. His ankle-brachial index was normal. The leukemia had been treated for 3 years with hydroxyurea, to a cumulative dose of $550 \mathrm{~g}$. A diagnosis of hydroxyurea-induced ulceration was made, and 8 weeks after the treatment with hydroxyurea was discontinued, the ulcers had resolved (Figure 2).

Hydroxyurea is a hydroxylated derivative of urea and has been recognized since 1960 as an effective chemotherapy agent for the treatment of some types of cancer. The most common indications for hydroxyurea therapy are chronic myeloid leukemia and other myeloproliferative disorders.

Adverse effects are usually mild and include fatigue, headache, nausea, vomiting, diarrhea and fever. Dermatologic side effects can also occur, including alopecia, diffuse hyperpigmentation, discoloration of the nails, stomatitis or dermatomyositis.

Ulcers of the leg occur in about $9 \%$ of patients who are taking high-dose, long-term hydroxyurea therapy for myeloproliferative diseases. ${ }^{1}$ The ulcers are painful and are often localized to the malleolar region. They are usually erythematous and surrounded by atrophie blanche (white stel-

From the Department of Dermatology, Faculty of Medicine, University of Duisburg-Essen, Essen, Germany

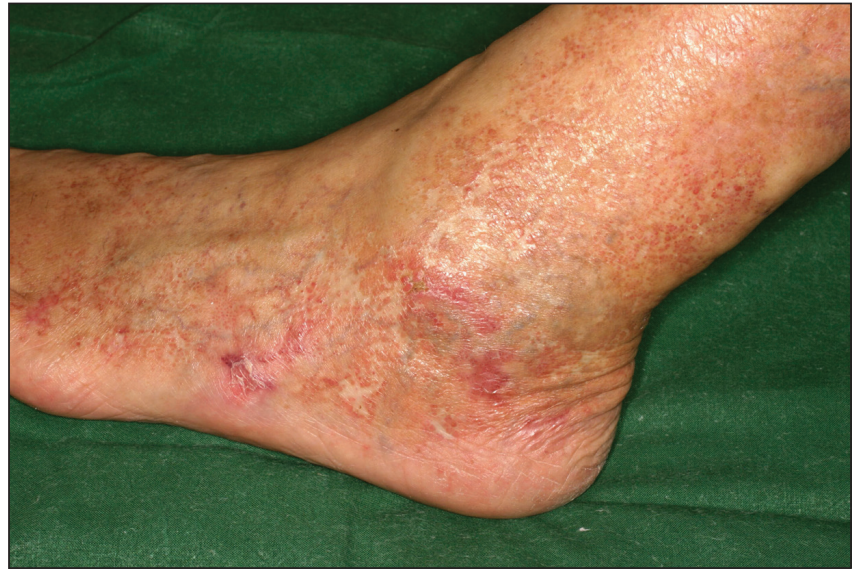

Figure 2: Resolution of the ulcers 8 weeks after hydroxyurea therapy was discontinued.

late scarring). Histopathologic analysis shows epidermal atrophy, dermal fibrosis and scar tissue without vascular lesions. ${ }^{1}$

The ulcers are caused by a reduction in cell viability that occurs when hydroxyurea disrupts the $\mathrm{S}$ phase (synthesis) of the cell cycle. Hydroxyurea inhibits the synthesis of DNA, which causes damage to basal keratinocytes and hinders the production of collagen. Inflammatory mediators that are derived from platelets and related to myeloproliferative disorders may play a role in causing the ulcers. Treatment involves stopping the hydroxyurea therapy and applying a moist wound dressing. The ulcers do not respond to conventional therapy alone and will not heal without cessation of hydroxyurea.

\section{REFERENCE}

1. Sirieix ME, Debure C, Baudot N, et al. Leg ulcers and hydroxyurea: forty-one cases. Arch Dermatol 1999;135:818-20.

Clinical images are chosen because they are particularly intriguing, classic or dramatic. Submissions of clear, appropriately labelled high-resolution images must be accompanied by a figure caption and the patient's written consent for publication. A brief explanation (300 words maximum) of the educational significance of the images with minimal references is required. 